\title{
Influência do ciclo lunar na captura de morcegos Phyllostomidae
}

\author{
Carlos E. L. Esbérard
}

Instituto de Biologia, Universidade Federal Rural do Estado do Rio de Janeiro, km 47 da antiga estrada Rio - São Paulo, Caixa Postal 74503, 23851-970 Seropédica, Rio de Janeiro, Brasil. (cesberard@ufrrj.br)

\begin{abstract}
Influence of moon cycle in phyllostomid bat capture. The influence of moonlight on the activity patterns of bats has been reported to some species. In general, bats react to the increase of nocturnal illumination by reducing the use of open spaces, by limiting foraging activity or by shortening the activity periods. To determine the influence of moon cycle on the efficiency of bat netting, captures of 28 night-samplings were analyzed in southeastern Brazil. The capture rate and species richness were inversely related to the moonlight intensity. Among the most frequently netted species, only two showed significant and negative relation with increasing moonlight. The highest variation in species richness was observed in gleaners and nectarivorous species. The days following new moon were the most productive in terms of capture rate and species richness. The local species richness was not archived when sampling was restricted to any single period of the moon cycle. Bat community studies may benefit from samplings spanning the entire moon cycle.
\end{abstract}

KEYWORDS. Lunar phobia, sampling methods, southeastern Brazil, bats.

RESUMO. A influência da luz da lua no padrão de atividade de morcegos já foi documentada em algumas espécies. Geralmente os morcegos reagem ao aumento da iluminação reduzindo o uso de espaços abertos e restringindo a atividade de forrageio ou a duração do período de atividade. Para estabelecer a influência do ciclo lunar na eficiência de captura de morcegos, foi realizada uma análise com 28 noites de capturas no sudeste do Brasil. A taxa de captura e a riqueza de espécies apresentaram relação linear inversa com a porção iluminada pela lua. Das espécies mais freqüentemente capturadas, apenas duas apresentaram relação significativa e negativa com o aumento da iluminação pela lua. Maiores variações na riqueza de espécies foram observadas em morcegos catadores e nectarívoros. Os dias após a lua nova são os mais produtivos em relação a capturas e riqueza. A riqueza local não pode ser totalmente amostrada se a amostragem for restrita a qualquer período do ciclo lunar. Para estudar a comunidade de morcegos é mais apropriado realizar amostragem durante todo o ciclo lunar.

PALAVRAS-CHAVE. Fobia lunar, métodos de amostragem, sudeste do Brasil, morcegos.

A influência da iluminação da lua já foi analisada em diversos grupos de mamíferos noturnos, como marsupiais, roedores, lagomorfos, carnívoros, morcegos e primatas (JULIEN-LAFERRIÈRE, 1997). O comportamento de evitar áreas abertas sob alta luminosidade lunar ou restringir a atividade a períodos mais escuros tem sido chamado de fobia lunar (MORRISON, 1978; SingARAVELAN \& MARImuthu, 2002). A maioria das espécies apresenta fobia lunar, reduzindo seu período total de atividade, concentrando suas atividades em períodos onde a luminosidade da lua mostra-se menos intensa (GURSKY, 2003) ou optando por permanecer e por deslocar-se em áreas com densa cobertura (Reith, 1982; Singaravelan $\&$ MARIMUTHU, 2002).

A fobia lunar já foi confirmada em espécies de morcegos frugívoros, como Artibeus jamaicensis Leach, 1821 (Morrison, 1978, 1980) e hematófagos, Desmodus rotundus (E. Geoffroy, 1810) (CRESPO et al., 1972). No entanto, morcegos insetívoros não alteraram o comportamento em noites com maior luminosidade (NegraefF \& Brigham, 1995; Hecker \& Brigham, 1999; KARLSSON et al., 2002) ou usaram locais mais sombreados (REITH, 1982).

Duas causas têm sido apontadas para a fobia lunar: (1) estratégia anti-predador (ERKERT, 1982; MORRISON, 1978, 1980) e (2) diminuição na densidade de possíveis presas (HeCKer \& BRIGHAM, 1999). A possibilidade de fobia lunar tem levado pesquisadores de morcegos a restringir as coletas às noites mais escuras do ciclo lunar com vistas a maximizar a eficiência de captura (REIS \& MULLER, 1995; ReIs et al., 1995).

O objetivo deste trabalho é (1) testar a influência do ciclo lunar sobre a freqüência local de morcegos Phyllostomidae analisando a taxa de captura de morcegos em diferentes fases do ciclo e (2) delinear a melhor estratégia de coleta.

\section{MATERIAL E MÉTODOS}

A Estação Ecológica Estadual Paraíso situa-se nos Municípios de Guapimirim e Cachoeiras de Macacu, Estado do Rio de Janeiro (4254’30,0”W; 22¹9'13,2”S, $80 \mathrm{~m}$ de altitude), a cerca de $90 \mathrm{~km}$ da cidade do Rio de Janeiro. Possui aproximadamente 4.920 ha e limita-se ao norte com o Parque Nacional da Serra dos Órgãos. Estende-se de 80 a $1.000 \mathrm{~m}$ de altitude. A área apresenta reduzida pressão antrópica, com extração seletiva de madeira observada até a década de 70 .

Os morcegos foram capturados com auxílio de redes de neblina abertas por toda a noite. Das 34 noites de coleta realizadas entre abril de 1997 e março de 1999, aquelas em que choveu (seis noites) foram eliminadas, pois a chuva é um dos fatores ambientais que interfere com a atividade dos morcegos, tanto pela diminuição de suas presas, como pela dificuldade de usar a ecolocalização (O'FARRELL, 1974; ERKERT, 1982). A cada noite, de 10 a 14 redes de neblina foram usadas e permaneceram nos mesmos locais, sendo duas ou três 
sobre riacho, cinco ou seis em trilhas previamente existentes e as demais em clareiras. As capturas foram uniformizadas pelo total de horas de esforço e pelo número de redes de neblina usadas a cada noite e expressas pela unidade capturas/horas*redes (MedELLín, 1993). Os morcegos capturados foram marcados com tatuagem no dactilopatágio e as recapturas observadas na mesma noite não foram consideradas na análise.

O ciclo lunar foi analisado através da percentagem da face iluminada (NEGRAEFF \& BRIGHAM, 1995), obtida através do programa Moontool for Windows 2.0. A diversidade em cada noite de coleta foi calculada pelo Índice de Shannon (MAGURRAN, 1988), usando o programa EcoSim 7.0. Testamos a relação entre o total da face iluminada da lua (variando de $0 \%$ na lua nova a $100 \%$ na lua cheia) com o total de capturas. A riqueza de espécies observada a cada noite de coleta e a diversidade de espécies foram analisadas através de uma relação linear simples. Cada espécie foi inserida em uma das guildas tróficas propostas por Kalko et al. (1996) e o total de espécies em cada guilda foi agrupado em classes quanto ao total iluminado da lua $(0-25 \%, 26-50 \%, 51-75 \%$ e $76-$ $100 \%$ ) para a análise da riqueza de espécies.

Para simular a melhor estratégia para definir as coletas ao longo do ciclo lunar, arbitrou-se a priori o total de 14 noites $(50 \%$ do total considerado neste trabalho) como ideal. Dez combinações aleatórias foram selecionadas para comparar com o total de espécies e capturas obtidas para as 14 noites mais próximas da lua nova, as 14 noites mais próximas da lua cheia e as 14 noites com os valores mais próximos de $50 \%$ da face iluminada.

Material testemunho das espécies está depositado na coleção de referência do Projeto Morcegos Urbanos (Processo 1755/89 - IBAMA/RJ), na Fundação RIOZOO: Artibeus obscurus Schinz, 1821 - PMU1617; Carollia perspicillata (Linnaeus, 1758) - PMU1670; Artibeus cinereus (Gervais, 1856) - PMU1674; Vampyressa pusilla (Wagner,
1843) - PMU1675; Artibeus lituratus (Olfers, 1818) PMU1679; Artibeus fimbriatus Gray, 1838 - PMU1681; Sturnira lilium (E. Geoffroy, 1810) - PMU1682; Platyrrhinus lineatus (E. Geoffroy, 1810) - PMU1684; Micronycteris hirsuta (Peters, 1869) - PMU1691; Trachops cirrhosus (Spix, 1823) - PMU1693; Lonchorhina aurita Tomes, 1863 - PMU1753; Anoura caudifer (E. Geoffroy, 1818) - PMU1756; D. rotundus (E. Geoffroy, 1810) - PMU1757; Tonatia bidens (Spix, 1823) - PMU1762; Micronycteris megalotis (Gray, 1842) - PMU1804; Pygoderma bilabiatum (Wagner, 1843) - PMU1867; Phylloderma stenopus Peters, 1865 - PMU1965; Lonchophylla mordax Thomas, 1903 - PMU2050; Diphylla ecaudata (Spix, 1823) - PMU2214; A. jamaicensis Leach, 1821 - PMU2239; Lonchophylla bokermanni Sazima, Vizotto \& Taddei, 1978 - PMU2331; Phyllostomus hastatus (Pallas, 1767) - PMU2459.

\section{RESULTADOS}

O total de 28 noites foi analisado (116.256 h-rede), sendo observadas 824 capturas e 22 espécies de Phyllostomidae. O total de capturas e a riqueza de espécies apresentaram relação negativa e significativa com a face iluminada da lua $(r=0,437, p=0,002 ; r=0,389, p=0,004$, respectivamente) (Figs. 1,2). Não verificou-se relação entre a diversidade de Shannon e a face iluminada da lua $(\mathrm{r}=0,065, \mathrm{p}=0,757)$.

As guildas de espécies frugívoras de dossel (6 a 7 espécies) e de sub-bosque ( 2 a 3 espécies) apresentaram menores variações no número de espécies que as guildas de nectarívoros ( 0 a 3 espécies) e catadores (1 a 4 espécies) nas diferentes classes adotadas (Fig. 3). Os nectarívoros apresentaram mais espécies nas noites mais escuras e os catadores nas noites mais claras.

A maior taxa de capturas foi obtida na fase de 26$50 \%$ da face iluminada e a menor em $75-100 \%$. O maior número de espécies foi observado com 76-100\% da face

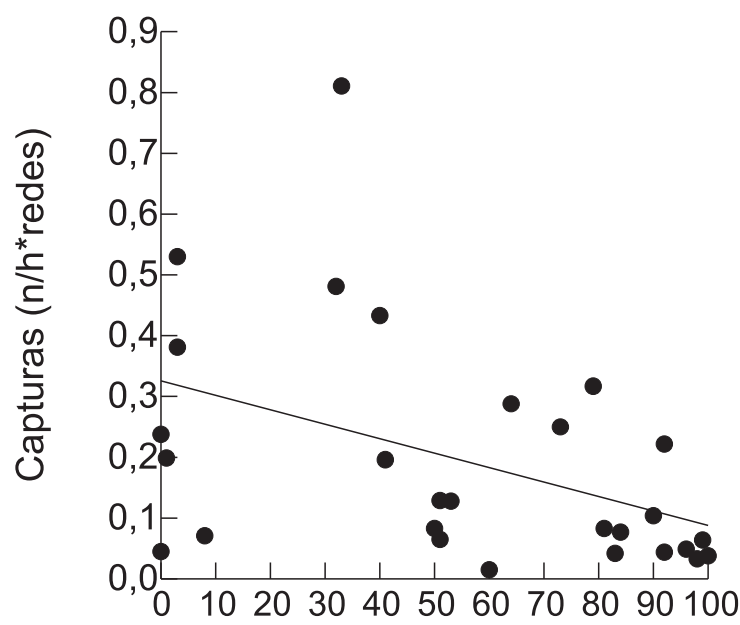

Face iluminada (\%)

Fig. 1. Variação da captura de morcegos em 28 noites com a porção iluminada da lua.

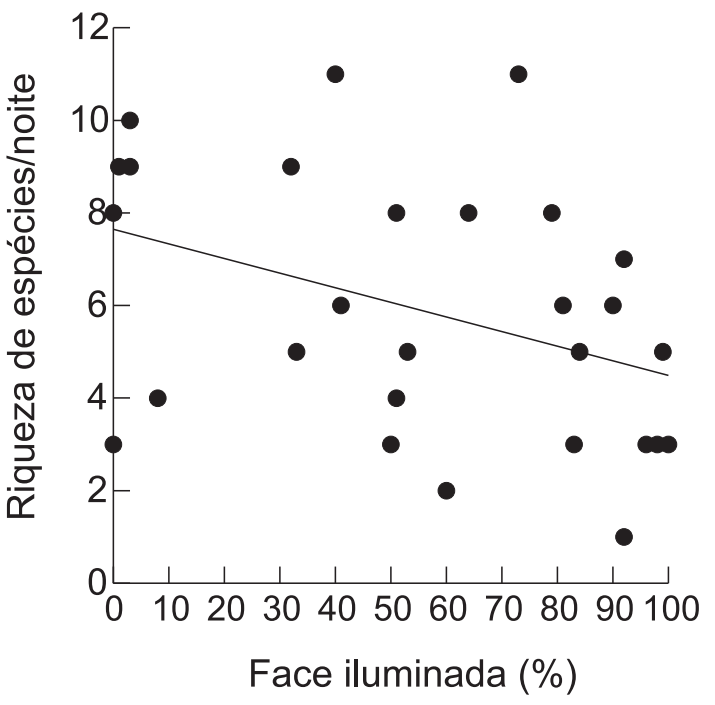

Fig. 2. Variação da riqueza de espécies em 28 noites com a porção iluminada da lua. 
iluminada e o menor número de espécies com 26 a $50 \%$ da face iluminada da lua (Tab. I). Das 10 espécies mais capturadas, duas apresentaram relação negativa e significativa com a face iluminada da lua - A. jamaicensis e $S$. lilium - e duas com valores muito próximos de serem significantes $-C$. perspicillata e A. lituratus (Tab. I).

O dendograma qualitativo utilizando o método de agrupamento UPGMA demonstrou que as classes intermediárias (26-50\% e 51-75\%) agrupam-se e separamse das classes extremas (0-25\% e 76-100\%) (Fig. 4).

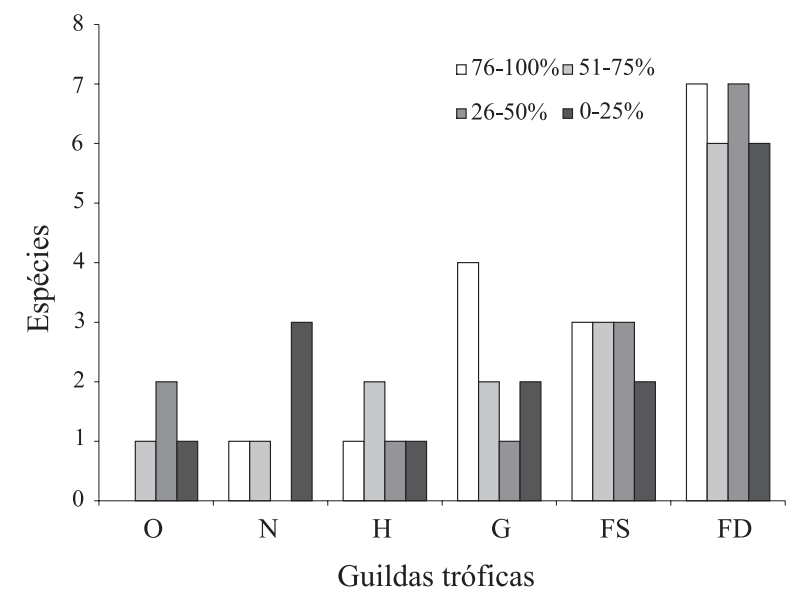

Fig. 3. Variação da riqueza de espécies em guildas tróficas (segundo KALKO et al., 1996) nas quatro semanas do ciclo lunar (FD, frugívoros de dossel; FS, frugívoros de sub-bosque; G, catadores; H, hematófagos; O, onívoros; N, nectarívoros).

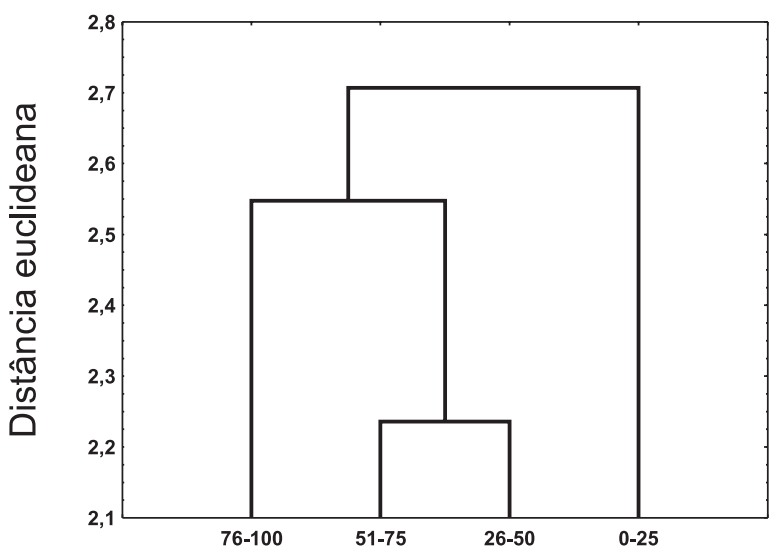

Fig. 4. Análise de agrupamento UPMGA qualitativo para as quatro semanas do ciclo lunar.

Tabela I. Taxa de captura para espécies de Phyllostomidae, guildas tróficas, diversidade de Shannon-Winner e valores da relação linear simples com a percentagem da face iluminada da lua para as 10 espécies mais freqüentemente capturadas (* relação significativa (p < 0,05); ** valores marginalmente significativos; CAT, catadores; FD, frugívoros de dossel; FSB, frugívoros de sub-bosque; HEM, hematófagos; NEC, nectarívoros; ONI, onívoros).

\begin{tabular}{|c|c|c|c|c|c|c|c|c|}
\hline \multirow[b]{2}{*}{ ESPÉCIES } & \multicolumn{7}{|c|}{ CAPTURAS (n/h*redes) } & \\
\hline & GUILDA & $0-25$ & $26-50$ & $51-75$ & $76-100$ & $\mathrm{r}$ & $\mathrm{p}$ & \\
\hline Artibeus obscurus Schinz, 1821 & FD & 0,0028 & 0,0381 & 0,0049 & 0,0012 & 0,22 & 0,26 & \\
\hline Carollia perspicillata (Linnaeus, 1758) & FSB & 0,0142 & 0,0124 & 0,0101 & 0,0044 & 0,37 & 0,06 & $* *$ \\
\hline Artibeus lituratus (Olfers, 1818) & FD & 0,0027 & 0,0102 & 0,0011 & 0,0008 & 0,36 & 0,06 & $* *$ \\
\hline Artibeus jamaicensis Leach, 1821 & FD & 0,0021 & 0,0076 & 0,0017 & 0,0004 & 0,47 & 0,01 & $*$ \\
\hline Sturnira lilium (E. Geoffroy, 1810) & FSB & 0,0014 & 0,0023 & 0,0009 & 0,0002 & 0,36 & 0,02 & $*$ \\
\hline Artibeus fimbriatus Gray, 1838 & FD & 0,0005 & 0,0031 & 0,0008 & 0,0002 & 0,28 & 0,16 & \\
\hline Artibeus cinereus (Gervais, 1856) & FD & 0,0009 & 0,0017 & 0,0002 & 0,0002 & 0,28 & 0,15 & \\
\hline Tonatia bidens (Spix, 1823) & CAT & 0,0001 & 0,0003 & 0,0021 & 0,0002 & 0,07 & 0,74 & \\
\hline Desmodus rotundus (E. Geoffroy, 1810) & HEM & 0,0002 & 0,0006 & 0,0009 & 0,0001 & 0,05 & 0,82 & \\
\hline Pygoderma bilabiatum (Wagner, 1843) & FSB & & 0,0008 & 0,0006 & 0,0002 & 0,14 & 0,49 & \\
\hline Phyllostomus hastatus (Pallas, 1767) & ONI & 0,0001 & 0,0006 & & & & & \\
\hline Anoura caudifer (E. Geoffroy, 1818) & NEC & 0,0002 & & 0,0004 & 0,0001 & & & \\
\hline Platyrrhinus lineatus (E. Geoffroy, 1810) & FD & 0,0001 & 0,0003 & 0,0002 & 0,0001 & & & \\
\hline Phylloderma stenopus Peters, 1865 & ONI & & 0,0003 & 0,0002 & & & & \\
\hline Vampyressa pusilla (Wagner, 1843) & FD & & 0,0003 & & 0,0001 & & & \\
\hline Trachops cirrhosus (Spix, 1823) & CAT & 0,0001 & & & 0,0002 & & & \\
\hline Lonchophylla mordax Thomas, 1903 & NEC & 0,0002 & & & & & & \\
\hline $\begin{array}{l}\text { Lonchophylla bokermanni } \\
\quad \text { Sazima, Vizotto \& Taddei, } 1978\end{array}$ & NEC & 0,0002 & & & & & & \\
\hline Lonchorhina aurita Tomes, 1863 & CAT & & & 0,0002 & & & & \\
\hline Diphylla ecaudata (Spix, 1823) & HEM & & & 0,0002 & & & & \\
\hline Micronycteris hirsuta (Peters, 1869) & CAT & & & & 0,0001 & & & \\
\hline Micronycteris megalotis (Gray, 1842) & CAT & & & & 0,0001 & & & \\
\hline TOTAL DE CAPTURAS & & 0,0285 & 0,0785 & 0,0244 & 0,0085 & & & \\
\hline DIVERSIDADE SHANNON-WINNER (H') & & 1,59 & 1,65 & 1,92 & 1,76 & & & \\
\hline
\end{tabular}



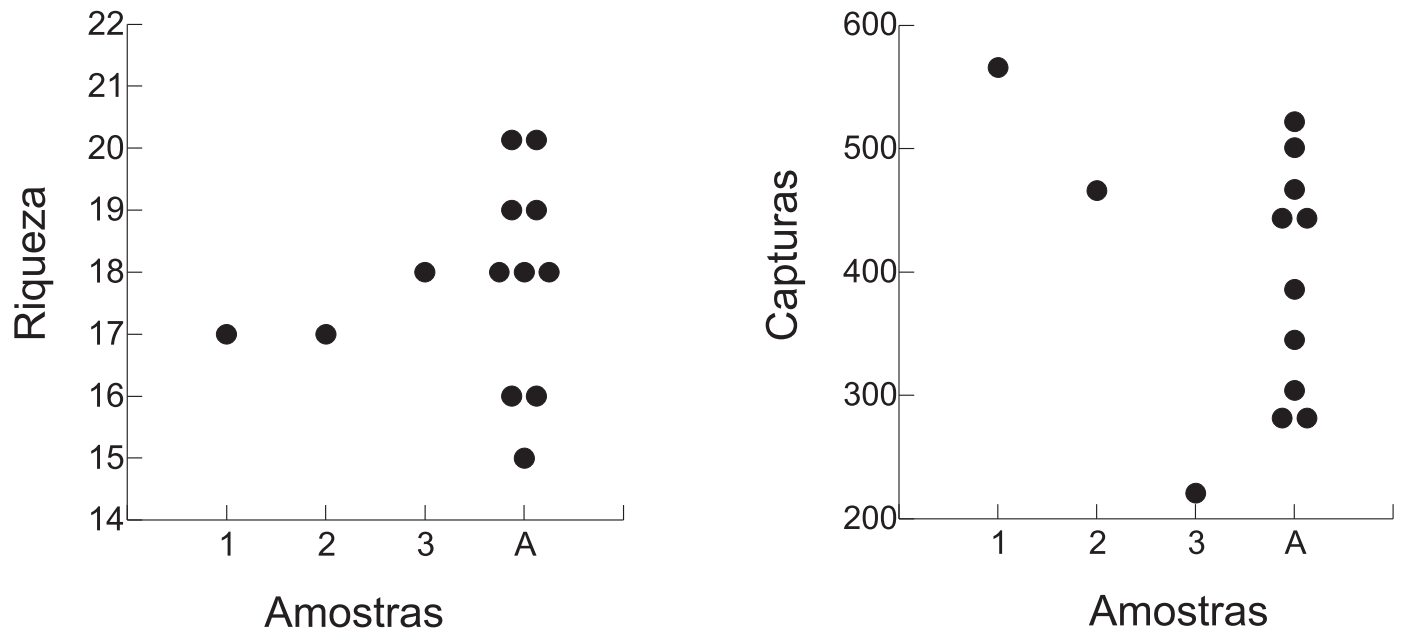

Fig. 5. Variação da riqueza (esquerda) e do total de capturas (direita) para 10 combinações aleatórias (A), para as 14 noites com menor face iluminada (1), para as 14 noites com face iluminada mais próxima de $50 \%$ (2) e para as 14 noites com maior face iluminada (3)

O total de espécies acumuladas se mostrou pouco mais elevado ( $\mathrm{N}=18$ espécies $)$ para as coletas escolhidas entre as mais próximas da lua cheia $(\overline{\mathrm{x}}=85,07 \% \pm 12,71 \mathrm{da}$ face iluminada) do que o observado para as coletas escolhidas entre as noites mais próximas da lua nova $(\overline{\mathrm{x}}=$ $26,14 \% \pm 22,22$ da face iluminada, $\mathrm{N}=17$ espécies) e das coletas com face iluminada próxima a $50 \%(\overline{\mathrm{x}}=56,57 \% \pm$ 17,50 da face iluminada, $\mathrm{N}=17$ espécies). As 10 combinações aleatórias resultaram em média de 18,18 \pm 1,88 espécies acumuladas, com $70 \%$ das combinações com riquezas igual ou superior a 18 espécies (Fig. 5). O total de espécies acumuladas mostrou-se independente do total de capturas $(\mathrm{r}=0,11, \mathrm{p}=0,73)$ e a maioria das combinações aleatórias resultaram em menos capturas que o total obtido com as 14 noites próximas à lua nova.

\section{DISCUSSÃO}

A acuidade visual de predadores noturnos, como as corujas, diminui com a redução do luar. Em baixa luminosidade o sucesso de ataque é muito reduzido e o tempo de procura e caça aumenta. Com isso, espera-se que a taxa de predação sobre morcegos e outros pequenos mamíferos noturnos seja aumentada em noites com maior luminosidade. É esperado que a atividade de pequenos mamíferos diminua com o aumento da iluminação noturna (KRAMER \& BIRNEY, 2001). A utilização de espaços abertos pelos pequenos mamíferos diminui em noites com maior influência do luar (JULIEN-LAFERRIÈRE, 1997).

Nem todas as espécies respondem da mesma forma à luminosidade, sendo a fobia lunar mais evidente na maior parte dos morcegos frugívoros e em uma espécie de hematófago já estudadas (CRESPO et al., 1972; MorRISON, 1978, 1980). Entretanto, neste trabalho, apenas quatro espécies apresentaram indícios de fobia lunar.

Uma guilda teve menos espécies capturadas na proximidade da lua nova - catadores, na qual insere-se a maioria das espécies de morcegos da subfamília Phyllostominae (KALKO et al., 1996). Estes morcegos são considerados como indicadores da qualidade do habitat (FEnTON et al., 1992). Em inventários realizados na Mata
Atlântica do Estado do Rio de Janeiro, esta subfamília foi representada por uma a seis espécies em cada localidade amostrada e cada espécie tem sido representada por um reduzido número de capturas (TEIXEIRA \& PERACCHI, 1996; DiAs et al., 2002; ESBÉRARD, 2003). A realização de coletas apenas em parte do ciclo lunar pode resultar em subamostragem deste táxon. A maior captura destas espécies em noites com maior porção da lua iluminada pode ser decorrente da estratégia alimentar por elas utilizada, pois se orientam pela visão, além do olfato e audição para a captura dos artrópodes e pequenos vertebrados (KALKO et al., 1996).

Alguns pesquisadores optaram em restringir suas coletas às semanas próximas à lua nova como meio de maximizar as capturas. Os dados obtidos neste trabalho mostram que as coletas mais próximas da lua nova são as mais produtivas no que se refere às capturas a cada noite. Foi observada relação negativa da taxa de captura nas quatro espécies mais freqüentemente capturadas no local (A. jamaicensis, S. lilium, C. perpspicillata e A. lituratus) e que representaram mais de $70 \%$ das capturas em coletas realizadas durante a lua nova. Menores proporções destas espécies são observadas em coletas realizadas nas demais fases do ciclo lunar, o que explica o maior sucesso quantitativo de coletas na lua nova. No entanto, coletas restritas a qualquer período do ciclo lunar provavelmente não amostrarão satisfatoriamente a riqueza total de Phyllostomidae do local. Em cada período analisado, correspondente a aumentos de $25 \%$ da face iluminada, de 67 a $76 \%$ do total de espécies encontradas no local foram amostradas. A simulação através de combinações aleatórias de coletas demonstrou que em $70 \%$ dos casos seria esperado resultado igual ou melhor que o obtido com coletas realizadas apenas próximas à lua nova.

Vários outros fatores podem influenciar na taxa de captura de quirópteros, tais como vento, chuva e nebulosidade (LEE \& MCCRAKEN, 2001). É também provável que o horário de nascer e pôr da lua influencie parcialmente a atividade das espécies. A baixa variação da riqueza e do número de capturas com a variação da face iluminada da lua pode ser resultante destes fatores, não considerados nesta análise. 
Estudos ecológicos centrados na estrutura e funcionamento da comunidade de quirópteros devem diversificar as coletas dentro do ciclo lunar, evitando subamostrar a riqueza e capturar em maior número as espécies que apresentam fobia lunar.

Agradecimentos. Ao Dr. Alcides Pissinatti, da Estação Ecológica Estadual Paraíso, pela permissão para coleta e o apoio concedido. Eliane M. Luz, Alexandre S. Chagas, Rodrigo Costa, Rodrigo Carneiro, Roberta Cruz, Mariane S. Nunes, Luciana F. S. Martins e Eduardo Rubião auxiliaram no trabalho de campo. Agradeço as críticas de Adarene Guimarães Motta e da Dra. Helena G. Bergallo. Dra. Cristina A. G. Nassar auxiliou na elaboração do abstract. Dr. Adriano L. Peracchi confirmou a identificação de parte do material testemunho das espécies coletadas. Este trabalho foi realizado sob a licença especial para coleta (Processo 1785/ 89-IBAMA). O autor agradece a bolsa de CNPq concedida (Processo 151029/2004-0).

\section{REFERÊNCIAS}

Crespo, R. F.; Linhart, R. B.; Burns, R. J. \& Mitchell, G. C. 1972. Foraging behavior of the common Vampire bat related to moonlight. Journal of Mammalogy 53(2):366-368.

Dias, D.; Silva, S. S. P. \& Peracchi, A. L. 2002. Quirópteros do Parque Estadual da Pedra Branca, Rio de Janeiro, RJ (Mammalia: Chiroptera). Revista Brasileira de Zoologia 19(supl. 2):113-140.

ERKert, H. G. 1982. Ecological aspects of bat activity. In: Kunz, T. H. ed. Ecology of Bats. New York and London, Plenum. p. 201-242.

EsBÉRARD, C. E. L. 2003. Diversidade de morcegos em uma área de Mata Atlântica regenerada no sudeste do Brasil (Mammalia: Chiroptera). Revista Brasileira de Zoociências 5(2):189-204.

Fenton, M. B.; Acharya, L.; Audet, D.; Hickey, M. B. C.; Merriman, C.; Obrist, M. K.; Syme, D. M. \& Adkins, B. 1992. Phyllostomid bats (Chiroptera: Phyllostomidae) as indicators of habitat disruption in the Neotropics. Biotropica 24(3):440-446.

Gursky, S. 2003. Lunar philia in a nocturnal Primate. International Journal of Primatology 24(2):251-267.

Hecker, K. R. \& Brigham, R. M. 1999. Does moonlight change vertical stratification of activity by forest-dwelling insectivorous bats? Journal of Mammalogy 80(4):1196-1201.

Hutson, A. M.; Mickleburgh, S. P. \& Racey, P. A. 2001. Global status survey and conservation action plan. Microchiropteran bats. London, Information. 259p.
JuLIEN-LAFERRIÈre, D. 1997. The influence of moonlight on activity of wolly opossums (Caluromys philander). Journal of Mammalogy 78(1):251-255.

Kalko, E. K. V.; Handley, C. O., JR. \& Handley, D. 1996. Organization, diversity and long-term dynamics of a neotropical bat community. In: Cody, M. L. \& Smallwood, J. A. Long-term studies of vertebrate communities. San Diego, Academic. p.503-553.

Karlsson, B. L.; Ekllof, J. \& Rydell, J. 2002. No lunar phobia in swarming insectivorous bats (family Vespertilionidae). Journal of Zoology 256:473-477.

Kramer, K. M. \& Birney, E. C. 2001. Effect of light intensity on activity patterns of Patagonian leaf-eared mice, Phyllotis xanthopygus. Journal of Mammalogy 82(2):535-544.

Lee, Y. \& McCraken, G. F. 2001. Timing and variation in the emergence and return of Mexican free-tailed bats, Tadarida brasiliensis mexicana. Zoological Studies 40(4):309-316.

Magurran, A. E. 1988. Ecological diversity and its measurement. London, Croom Helm. 179p.

Medellín, R. A. 1993. Estructura y diversidad de una comunidad de murciélagos en el trópico húmedo mexicano. In: Medellín, R. A. \& Ceballos, T. G. eds. Avances en el estudio de los mamíferos de México. México, Asociación Mexicana de Mastozoología. v. 1, p.333-354.

Morrison, D. W. 1978. Foraging ecology and energetics of the frugivorous bat Artibeus jamaicensis. Ecology 59(4):716-723. . 1980. Foraging and day-roosting dynamics of canopy fruit bats in Panama. Journal of Mammalogy 61:20-29.

NegraefF, O. E. \& Brigham, R. M. 1995. The influence of moonlight on the activity of little brown bats (Myotis lucifugus). Zeischrift fur Säugetierkunde 60:330-336.

O'FARRELl, M. J. 1974. Seasonal acitivity patterns of rodents in an sagebrush community. Journal of Mammalogy 55:809-823.

Reis, N. R. \& Muller, M. F. 1995. Bat diversity of forests and open areas in a subtropical region of south Brazil. Ecologia Austral 5:31-36.

Reis, N. R.; Peracchi, A. L.; Muller, M. F.; Bastos, E. A. \& Soares, E. S. 1995. Quirópteros do Parque Estadual do Morro do Diabo, São Paulo, Brasil (Mammalia: Chiroptera). Revista Brasileira de Biologia 56(1):87-92.

Reith, C. C. 1982. Insectivorous bats fly in shadows to avoid moonlight. Journal of Mammalogy 63(4):685-688.

Singaravelan, N. \& Marimuthu, G. 2002. Moonlight inhibits and lunar eclipse enhances foraging activity of fruit bats in an orchad. Current Science 82(8):1020-1022.

Teixeira, S. C. \& Peracchi, A. L. 1996. Morcegos do Parque Estadual da Serra da Tiririca, Rio de Janeiro, Brasil (Mammalia, Chiroptera). Revista Brasileira de Zoologia 13:61-66.

Recebido em fevereiro de 2005. Aceito em setembro de 2006. ISSN 0073-4721

Artigo disponível em: www.scielo.br/isz

Iheringia, Sér. Zool., Porto Alegre, 97(1):81-85, 30 de março de 2007 